\title{
DESIGNING A WATER QUALITY INDEX FOR KESBEWA LAKE
}

\author{
HS Wijetunge and S Hewage \\ Centre for Analytical Research and Development, Department of Chemistry, \\ University of Colombo, Colombo 03
}

Water Quality Index (WQI) is a rat nn reflecting the composite influence on overall quality of a number of individual quality parameters in a selected water body. The objective of this research project is to establish a comprehersive Water Quality Index (WQI) for the Kesbewa Lake by using physical, chemical and microbiological water quality parameters, to identify the pollution levels. These results could be use to maintain the quality of water, and conserve flora and fauna, and also to advise the individuals, organizations and finding bodies associated with the lake.

The Water Quality Index is definei as, WQI $=\sum$ WiQi where, WQI = Water Quality Index, a number between $0-100, Q i=$ Quality of the $i^{\text {th }}$ parameter, $W i=$ The weighting factor of the $i^{\text {th }}$ parameter, a number between 1 and $)$, such that, $\sum \mathrm{Wi}=1$ for $\mathrm{n}$ number of parameters, $\mathrm{Wi}=\mathrm{Xi}$ / $\mathrm{Y}, \mathrm{Xi}=$ Points given by reference material data for $\mathrm{i}^{\text {th }}$ parameter, $\mathrm{Y}=$ The total points for $\mathrm{n}$ number of parameters.

The raw analytical results for exch parameter, having different units of measurements, are transformed into unit less Q-values by using the respective function of quality value of each parameter in the Q-value graphs. These Q- value graphs are plotted with respect to the each measured parameter value and their relative $\mathrm{Q}$-values assigned by a points system.

Based on the World Health Organization (WHO) guidelines, points system for the Q-values for a parameter is assigned and Central Environment Authority (CEA) standards were used, where the WHO guidelines are not available. The highest Q-value 100 is assigned for the best value of a particular parameter that falls within the above guideline values. Separate ratings for Quality values were given for drinking and bathing, irrigation water, and for fish and aquatic life. These three Quality values were averagec to one value, which gives the Q-value for the measured value of that particular parameter in the Q-value graph. By using the functions of these graphs, the $\mathrm{Q}$-value (Qi) for any measured value of $i^{\text {th }}$ parameter can be obtained.

The weighting factor, (Wi) was determined by considering a large number of reference material data obtained from different water quality indices, in which the relative contribution of a parameter for the overall water quaiity has been weighted in different manner, according to the different point of views of scientists. The Q-value (Qi) is then multiplied by the weighting factor (Wi) and resulted values of all $n$ number of parameters are summed to yield the total value of the WQI.

Twenty samples were collected fror ten different locations of the lake over a period of eight months. According to the analyzed results, the prepared WQI for the Kesbewa lake gets a general ratings of 49.74 , which falls, in the region of Bad in the water quality index. In comparing the average values of measured parameters with WHO and Sri Lankan Standards, it can be concluded that the water of Kesbewa Lake is relatively polluted and water quality should be improved for aquatic life, irrigation and drinking and bathing purposes. 